\title{
Influence of femoral implant design modification on anterior knee pain and patellar crepitus in patients who underwent total knee arthroplasty without patella resurfacing
}

\author{
Yi-Fan Huang, Yu-Hang Gao, Lu Ding, Bo Liu, Jian-Guo Liu and Xin Qi
}

\begin{abstract}
Background: The incidence of patient dissatisfaction due to anterior knee pain (AKP) and patellar crepitus after total knee arthroplasty (TKA) remains a concern. However, it has been shown that improvements in the femoral component of traditional prostheses could reduce these instances of pain in the case of TKA performed with patellar resurfacing. This study aims to investigate whether TKA without patellar resurfacing can also benefit from the aforementioned femoral component modification in reducing AKP and patellar crepitus post-TKA.

Methods: Sixty-two patients (85 knees) who underwent TKA using the modern prosthesis and 62 age- and sexmatched patients (90 knees) fitted with the traditional prosthesis were enrolled in this study. The occurrence of AKP and patellar crepitus as well as the Knee Society Score (KSS) were consequently recorded, and the data was analyzed in order to determine whether there was a statistically significant difference between the two groups.

Results: The incidence of AKP was significantly lower in the study group compared with the control group at the 3-month and 1-year follow-ups (4.7\% vs. $13.3 \%[p=0.048]$ and 3.5\% vs. $13.3 \%[p=0.021]$, respectively). In addition, the incidence of patellar crepitus was also significantly lower in the study group compared with the control group at the 3-month and 1-year follow-ups (15.3\% vs. 34.4\% [ $p=0.004]$ and $10.6 \%$ vs. $28.9 \%$ [ $p=0.002]$, respectively). There was no significant difference in the KSS between the two groups.

Conclusions: These results revealed that TKA without patellar resurfacing will indeed benefit from the modified femoral implant design in reducing AKP and patellar crepitus, a finding that may be beneficial to surgeons who select implants for their patients when patellar resurfacing is not planned or not possible due to other reasons.
\end{abstract}

Keywords: Total knee arthroplasty, Femoral component, Prosthesis design, Anterior knee pain, Patellar crepitus

\footnotetext{
* Correspondence: qixindoc@163.com

Department of Orthopaedic Surgery, The First Hospital of Jilin University,

Changchun, Jilin 130021, China
}

(c) The Author(s). 2020 Open Access This article is licensed under a Creative Commons Attribution 4.0 International License, which permits use, sharing, adaptation, distribution and reproduction in any medium or format, as long as you give appropriate credit to the original author(s) and the source, provide a link to the Creative Commons licence, and indicate if changes were made. The images or other third party material in this article are included in the article's Creative Commons licence, unless indicated otherwise in a credit line to the material. If material is not included in the article's Creative Commons licence and your intended use is not permitted by statutory regulation or exceeds the permitted use, you will need to obtain permission directly from the copyright holder. To view a copy of this licence, visit http://creativecommons.org/licenses/by/4.0/. The Creative Commons Public Domain Dedication waiver (http://creativecommons.org/publicdomain/zero/1.0/) applies to the data made available in this article, unless otherwise stated in a credit line to the data. 


\section{Background}

Although good clinical outcomes have been achieved in patients undergoing total knee arthroplasty (TKA), the incidence of patient dissatisfaction due to multiple factors, especially anterior knee pain (AKP) and patellar crepitus after TKA, remains a concern [1-3]. The etiology and pathogenesis of AKP after TKA remain unclear, although several contributing factors have been identified, like patellar instability and maltracking $[4,5]$. Patellar crepitus is defined as a grinding sensation in the region of the distal quadriceps tendon over the patella when the knee is brought from flexion to extension, and secondary to fibrosynovial proliferation on the posterior aspect of the distal quadriceps tendon [6]. A previous study reported that up to one-third of patients who underwent TKA experienced mild to moderate AKP at the 1-year follow-up [7]. The incidence of patellar crepitus in posterior stabilized TKA can reach $45 \%$, which was significantly higher than in other types of prosthesis procedures [8]. Currently, new prostheses have incorporated modifications in the femoral component, with the aim of reducing complications, especially AKP and patellar crepitus. The main modifications of the new prostheses incorporate a new design of the femoral component that is more "friendly" to the patella, including a deeper and more extensive femoral trochlear groove and a smoother intercondylar box transition zone [9]. Recent clinical studies have shown that the femoral implant design modification in the ATTUNE Knee System (DePuy, Inc., Warsaw, IN, USA) decreases the incidence of AKP and patellar crepitus in posterior stabilized TKA $[10,11]$. Carey et al. even suggested that a significant difference was demonstrated in the 6 month postoperative clinical outcome between the P.F.C. (DePuy, Inc., Warsaw, IN, USA) and ATTUNE Knee Systems in patients who underwent TKA with both prostheses [12]. However, these studies did not enroll patients who underwent TKA without patellar resurfacing. Although many studies have suggested there is no significant difference in functional outcomes between TKA procedures that incorporated patellar resurfacing and that those that did not [13-15], the probability of AKP was higher in TKA procedures performed without patellar resurfacing than in those that were [16]. Whether TKA without patellar resurfacing benefits from this femoral implant design modification with regard to AKP and patellar crepitus remained unclear. The purpose of the present study, therefore, was to compare the incidence of AKP and patellar crepitus between patients who underwent TKA without patellar resurfacing using either the P.F.C. Sigma (DePuy Synthes) or the ATTU NE Knee Systems. Follow up data were taken at the 3 month and 1 year mark, postoperatively.

\section{Materials and methods}

\section{Study design}

A prospective, nested case-control study was performed in the authors' department, in accordance with the principles of the Helsinki Declaration. The Institutional
Review Board (IRB) approved the study (IRB number IRB00008484), and all patients provided informed consent for the treatment and publication of their anonymized data.

\section{Participants}

All patients scheduled for primary TKA for end-stage knee osteoarthritis between August 2016 and July 2017 were enrolled in this study. Individuals with constrained implant(s), revision, infection, postoperative disability caused by other serious diseases, and those lost in followup were excluded. Patients using the modern prosthesis system (i.e., ATTUNE) were selected as the study group. In creating the control group, we matched each study patient (ATTUNE group) with a patient using the traditional prosthesis (i.e., P.F.C. Sigma) for age ( \pm 3 years) and gender. Both prostheses are fixed bearing posterior stabilized total knee prostheses. Data on demographic characteristics and Knee Society Score (KSS) [17] were collected preoperatively for all participants.

A single senior surgeon performed all TKAs using a medial parapatellar approach. The anterior and posterior cruciate ligaments were removed. After proximal tibial and distal femoral bone cutting, a spacer was used to evaluate the extension gap to obtain a rectangular and equal extension gap. A stepwise release strategy was applied according to the tension of the soft tissue and ligaments. If the imbalance persisted, a subperiosteal peel method was used to further release. Femoral component rotation was determined using the gap balancing method. In $90^{\circ}$ flexion, a tension balancer that rotated on the axis of the femoral medullary canal was placed, and a line was drawn on the posterior femur that created a rectangular flexion gap of the same thickness as the extension gap. External femoral osteotomy was performed according to this gap balance line. An extramedullary device was used to determine the rotation of the tibial component. The proximal anatomical marker was the line connecting the medial border of the tibial tuberosity with the center of the posterior cruciate ligament, and the distal marker was the second metatarsal. The patella was reshaped to remove osteophytes. All knees exhibited good patellar tracking. In addition, patellar cartilage defect(s) were graded intraoperatively according to previous literature reports [18]. An identical postoperative rehabilitation program was scheduled for all patients.

Patients were evaluated at 3 months and 1 year after surgery, with radiographs acquired during each evaluation. KSS scores, AKP, and patellar crepitus were recorded by an investigator who was blinded to the prosthesis type. AKP was diagnosed if pain in the front of the knee was reported, and the degree of pain was scored using a visual analog scale (VAS), as follows: 0 or 
$1=$ no pain; 2 or $3=$ mild pain; $4-6=$ moderate pain; 7 or $8=$ severe pain; and 9 or $10=$ excruciating pain. A closed-chain weight-bearing exercise was used, and a series of further questions was administered to those who experienced AKP, as follows: During which of the following actions do you feel pain?: at rest; walking on flat ground; ascending and descending stairs; and squatting. The investigator diagnosed patellar crepitus by placing his hand on the patient's peripatellar region during full extension to flexion.

\section{Statistical methods}

Data from the two groups were analyzed statistically. Data normality was evaluated using the Kolmogorov-Smirnov test. Independent samples $t$-tests were applied to normally distributed values, while data with non-Gaussian distribution were analyzed using the non-parametric MannWhitney $U$ test. The observed frequencies of categorical variables were assessed using Pearson's chi-squared test. Descriptive statistics are expressed as mean with standard deviation and frequencies with percentages for categorical variables. All statistical analyses were performed using SPSS version 19 (IBM Corporation, Armonk, NY, USA), and differences with a $p$ value $<0.05$ were considered to be statistically significant.

\section{Results}

During the recruitment period, 202 consecutive patients were approached. There were 6 with constrained implant for severe varus or valgus deformity, 1 who died of unrelated disease, and 15 who were lost during followup that were excluded. Therefore, 180 patients were eligible for enrollment, of whom 62 (85 knees) were selected for the study group (23 were bilateral and 39 were unilateral involvements). From the remaining 118 patients, 62 (90 knees) age-matched and sex-matched patients were selected to afford a 1:1 match as the control group. Of these 62 patients, 28 were bilateral and 34 were unilateral involvements.

Demographic data and patellar cartilage grades are summarized in Table 1. There was no significant difference in age $(p=0.516)$, side $(p=0.362)$, body mass index $(p=0.878)$ or patellar cartilage grade $(p=0.670)$ between the two groups. In addition, no evidence of prosthesis loosening or osteolysis was found on postoperative radiographs.

There was no significant differences in clinical and functional scores between the study and control groups before surgery, at 3-months, or at the 1-year follow-up (Table 2).

The incidence of AKP was significantly lower in the study group compared with the control group at the 3month and 1-year follow-ups. Similarly, the incidence of patellar crepitus was significantly lower in the study
Table 1 Patient demographics and patellar cartilage grade

\begin{tabular}{llll}
\hline Measure & Control Group & Study Group & $p$ value \\
\hline Patients & 62 & 62 & - \\
Knees & 90 & 85 & - \\
Gender & & & - \\
$\quad$ Male & 10 & 10 & \\
$\quad$ Female & 52 & 52 & $0.362^{\mathrm{a}}$ \\
Side & & & \\
$\quad$ unilateral & 34 & 39 & \\
$\quad$ bilateral & 28 & 23 & $0.516^{\mathrm{b}}$ \\
Age & $64.98 \pm 4.79$ & $65.06 \pm 4.42$ & $0.878^{\mathrm{c}}$ \\
BMl & $26.13 \pm 2.78$ & $25.81 \pm 2.82$ & $0.226^{\mathrm{c}}$ \\
Follow-up & $1.0 \pm 0.44(0.9-1.1)$ & $1.0 \pm 0.53(0.9-1.1)$ & $0.670^{\mathrm{c}}$ \\
Patellar cartilage grade & $2.16 \pm 0.729$ & $2.26 \pm 0.676$ & \\
\hline
\end{tabular}

apearson Chi-squared test

b Independent-samples t-test

${ }^{\mathrm{c}}$ Non-parametric Mann-Whitney U test

group compared with that in the control group at the 3month and 1-year follow-ups (Table 3).

No new-onset AKP occurred at 1 year; however, 1 symptomatic knee that underwent TKA with the ATTU NE Knee System became asymptomatic at this time. In the control group, 12 patients reported AKP when squatting, among which 8 reported AKP during ascending and descending stairs. In the study group, 3 knees exhibited AKP when squatting, of which 2 exhibited AKP when ascending and descending stairs (Table 3). Two knees with PFC TKA experienced moderate AKP, which persisted at 1 -year postoperatively, the remainder of the cases experienced mild AKP at this time (Table 4).

\section{Discussion}

The question of whether or not to resurface the patella in TKA remains controversial [19]. A cost-effectiveness analysis demonstrated the superiority of resurfacing over retention of the patella [20]. On the other hand, selectively not resurfacing the patella appears to yield similar

Table 2 Preoperative and Postoperative KS clinical and function scores between the two groups

\begin{tabular}{llll}
\hline Measure & Control Group & Study Group & $p$ value \\
\hline Clinical scores & & & \\
Preoperatively & $56.81 \pm 12.58$ & $56.13 \pm 13.67$ & $0.286^{\mathrm{a}}$ \\
3-m Postoperatively & $86.65 \pm 7.43$ & $87.77 \pm 7.43$ & $0.855^{\mathrm{a}}$ \\
1-y Postoperatively & $90.24 \pm 7.68$ & $91.19 \pm 6.84$ & $0.380^{\mathrm{a}}$ \\
Function scores & & & \\
Preoperatively & $34.03 \pm 11.83$ & $35.24 \pm 12.03$ & $0.883^{\mathrm{a}}$ \\
3-m Postoperatively & $80.81 \pm 10.64$ & $82.34 \pm 11.08$ & $0.501^{\mathrm{a}}$ \\
1-y Postoperatively & $87.10 \pm 8.02$ & $88.55 \pm 8.70$ & $0.825^{\mathrm{a}}$
\end{tabular}

${ }^{\mathrm{a} N o n-p a r a m e t r i c ~ M a n n-W h i t n e y} \mathrm{U}$ test 
Table 3 Incidence of anterior knee pain, and patellar crepitus between the two groups

\begin{tabular}{|c|c|c|c|}
\hline Measure & Control Group & Study Group & $p$ value \\
\hline \multicolumn{4}{|l|}{ Anterior knee pain } \\
\hline 3-m Postoperatively & $12(13.3 \%)$ & $4(4.7 \%)$ & $0.048^{a}$ \\
\hline 1-y Postoperatively & $12(13.3 \%)$ & $3(3.5 \%)$ & $0.021^{a}$ \\
\hline \multicolumn{4}{|l|}{ Patellar creptius } \\
\hline 3-m Postoperatively & $31(34.4 \%)$ & $13(15.3 \%)$ & $0.004^{a}$ \\
\hline 1-y Postoperatively & $26(28.9 \%)$ & $9(10.6 \%)$ & $0.002^{\mathrm{a}}$ \\
\hline \multicolumn{4}{|c|}{ During which of the following actions do you feel pain? } \\
\hline Rest & 0 & 0 & \\
\hline Walking on flat & 0 & 0 & \\
\hline Ascending and descending stairs & 8 & 3 & \\
\hline Squatting & 12 & 2 & \\
\hline
\end{tabular}

${ }^{a}$ Pearson Chi-squared test

results compared with routine resurfacing [21]. The main feature of the modern prosthesis is the modification of the femoral component, which includes a deeper and more extensive femoral trochlear groove and a smoother intercondylar box transition zone. The new design aims-at least theoretically-to reduce the incidence of patellar crepitus and AKP. Previous research investigating the advantages of the femoral implant design modification only recruited patients who underwent TKA with patellar resurfacing [10-12]. To our knowledge, the present investigation was the first prospective study to compare the incidence of AKP and patellar crepitus in patients who underwent TKA without patellar resurfacing.

A meta-analysis showed that there was no relationship between cartilage condition and AKP [22]. Because this study did not resurface the patella and Vahur Metsna et al. [18] found that the cartilage damage to the patella may be correlated with postoperative AKP, we also matched patellar cartilage grade between our two groups. The clinical outcomes measured according to KSS at the 1-year follow-up were consistent with the outcomes reported by Ranawat et al. [11]. Carey et al. [12] suggested better outcomes at the 6-month followup; however, we did not find differences at the 3-month and 1-year follow-ups, which may have been due the different measured scores in the study by Carey et al. (i.e., Western Ontario and McMaster Universities Osteoarthritis Index, Oxford Knee, and Short-form-12 scores), or simply a temporary advantage existed at this period but disappeared at 1 year. Nevertheless, these results may indicate that this femoral implant design modification had no significant influence on clinical outcomes of TKA with or without patellar resurfacing at the shortterm follow-up.

Results of this study indicated that the ATTUNE group had a lower incidence of AKP, similar to the study by Ranawat et al. [11]. However, AKP incidences of 3.5 and $13.5 \%$ (ATTUNE and P.F.C. Sigma Knee Systems, respectively) were obviously lower than the incidences of AKP reported by Ranawat et al. (12.5 and $25.8 \%$, respectively). Asian patients tends to exhibit a longer duration of knee osteoarthritis. Because prolonged pain often results in an increase in pain tolerance, Asian patients may not be as sensitive to pain as those in developed countries. In previous research involving Japanese patients who underwent TKA, the incidence of AKP was also low (6.5\%) [23]. In addition, the incidence of AKP in the ATTUNE group was only approximately onequarter of that in P.F.C. Sigma group (3.5\% vs $13.5 \%)$ this ratio was lower than in the study by Ranawat et al. $(12.5 \%$ vs $25.8 \%)$ [11]. A previous study noted that

Table 4 VAS of anterior knee pain between the two groups

\begin{tabular}{|c|c|c|c|c|c|}
\hline Measure & none & mild & moderate & severe & excruciating \\
\hline \multicolumn{6}{|l|}{ Control Group } \\
\hline 3-m Postoperatively & 0 & $10(11.1 \%)$ & $2(2.2 \%)$ & 0 & 0 \\
\hline 1-y Postoperatively & 0 & $10(11.1 \%)$ & $2(2.2 \%)$ & 0 & 0 \\
\hline \multicolumn{6}{|l|}{ Study Group } \\
\hline 3-m Postoperatively & 0 & $4(4.7 \%)$ & 0 & 0 & 0 \\
\hline 1-y Postoperatively & 0 & $4(4.7 \%)$ & 0 & 0 & 0 \\
\hline
\end{tabular}


severe AKP can lead to patient dissatisfaction following primary TKA, and revision was often required [24]. In the present study, no patient underwent secondary patellar replacement. Furthermore, we recorded the state of movement when pain occurred for the assessment of AKP and found that it mainly occurred when ascending and/or descending stairs or squatting. It was rare for an individual to report pain while at rest or walking on flat ground. A sharp increase in pressure on the patella when ascending or descending stairs or squatting may be the underlying reason for a higher level of AKP. We assessed the degree of the pain using a VAS and found that two knees that underwent TKA using the PFC Sigma Knee Systems experienced moderate pain, which had a certain degree of impact on quality of life. In contrast, no patients using the ATTUNE Knee System experienced pain. The remainder of patients who experienced pain in our study reported it to be mild at the 2-year follow-up and it had little impact on the quality of life. These results may indicate that TKA without patellar resurfacing benefits from this femoral implant design modification with regard to AKP.

In the present study, the incidence of patellar crepitus at 1 year (10.6 and $28.9 \%$ in ATTUNE and P.F.C. Sigma groups, respectively) was similar to the incidences reported by Ranawat et al. (17.7 and $30.9 \%$, respectively) [11]. In addition, we found that the patellar crepitus occurred at 3 months, postoperatively. A previous analysis of posterior-stabilized TKAs revealed that the mean time to the diagnosis of patellar crepitus was approximately 10 months [25], which may suggest that patellar crepitus occurred at an earlier stage in TKA without patellar resurfacing. However, it does not affect the quality of life of patients given the level of reported satisfaction.

We acknowledge that the present study had several limitations. First, it was not blinded and randomized, so bias cannot be excluded as a confounding variable. However, we managed to prospectively match demographic data between cases and controls. Second, the sample size was calculated using a power analysis because no published data regarding TKA without patellar resurfacing were available for this study. Third, we did not enroll patients who underwent TKA with patellar resurfacing. Future studies should compare AKP and patellar crepitus and include both patients who undergo TKA with or without patellar resurfacing in the same study. Some have suggested that surgical technique, more size options, and rotation of the femoral component may also lead to these differences. As such, further research investigating these factors should be performed [26].

\section{Conclusions}

Modified femoral design is able to reduce postoperative anterior knee pain and crepitus in patients without patellar resurfacing. The finding of the current study is helpful for surgeons to select implants for their patients when patellar resurfacing is not planned or not possible due to other reasons.

\section{Abbreviations \\ TKA: Total Knee Arthroplasty; AKP: Anterior Knee Pain; KSSs: Knee Society Scores; VAS: Visual Analog Pain Scores}

\section{Acknowledgements \\ We would like to thank Cathy Lv and Editage (www.editage.cn) for English language editing.}

\section{Authors' contributions}

$X Q$ and JGL participated in the design of this study. YFH, LD and BL carried out the studies and performed the statistical analysis. YFH and YHG drafted the manuscript. All authors read and approved the final manuscript.

\section{Funding}

This study was supported by the grant (20170414020GH) from Department of Science and Technology of Jilin Province.

\section{Availability of data and materials}

The datasets used and/or analysed during the current study are available from the corresponding author on reasonable request.

\section{Ethics approval and consent to participate}

All procedures performed in the study were in accordance with the ethical standards of the ethics committee of The First Hospital of Jilin University (IRB number IRB00008484) and national research committee and conformed to the 1964 Helsinki declaration and its subsequent amendments. Informed consent for this study was obtained from all patients by both written and verbal.

\section{Consent for publication}

Written informed consent for publication was obtained from all participants.

\section{Competing interests}

All authors declare that they have no conflict of interest.

Received: 10 September 2019 Accepted: 1 June 2020

Published online: 09 June 2020

\section{References}

1. Baker PN, van der Meulen JH, Lewsey J, Gregg PJ, et al. The role of pain and function in determining patient satisfaction after total knee replacement. Data from the National Joint Registry for England and Wales. J Bone Joint Surg Br. 2007;89:893-900.

2. Jacobs $C A$, Christensen $C P$, et al. Factors influencing patient satisfaction two to five years after primary total knee arthroplasty. J Arthroplast. 2014;29: 1189-91.

3. Jacobs CA, Christensen CP, Karthikeyan T, et al. Patient and intraoperative factors influencing satisfaction two to five years after primary total knee arthroplasty. J Arthroplast. 2014:29:1576-9.

4. Pongcharoen B, Yakampor T, Charoencholvanish K. Patellar tracking and anterior knee pain are similar after medial parapatellar and midvastus approaches in minimally invasive TKA. Clin Orthop Relat Res. 2013;471(5): 1654-60.

5. van Jonbergen H-PW, Reuver JM, Mutsaerts EL, Poolman RW. Determinants of anterior knee pain following total knee replacement: a systematic review. Knee Surg Sports Traumatol Arthrosc. 2014;22(3):478-99.

6. Hwang B-H, Nam C-H, Jung K-A, Ong A, Lee S-C. Is further treatment necessary for patellar crepitus after total knee Arthroplasty? Clin Orthop Relat Res. 2013;471(2):606-12.

7. Meftah M, Ranawat AS, Ranawat CS, et al. The natural history of anterior knee pain in 2 posterior-stabilized, modular total knee arthroplasty designs. J Arthroplast. 2011:26:1145-8.

8. Nam D, Barrack T, Nunley RM, Barrack RL, et al. What is the frequency of noise generation in modern knee Arthroplasty and is it associated with residual symptoms. Clin Orthop Relat Res. 2017;475:83-90. 
9. Saffarini M, Demey G, Nover L, Dejour D. Evolution of trochlear compartment geometry in total knee arthroplasty. Ann Transl Med. 2016; 4(1):7.

10. Martin JR, Jennings JM, Watters TS, Levy DL, McNabb DC, Dennis DA, et al. Femoral implant design modification decreases the incidence of patellar crepitus in Total knee Arthroplasty. J Arthroplast. 2017;32:1310-3.

11. Ranawat CS, White PB, West S, Ranawat AS, et al. Clinical and radiographic results of Attune and PFC sigma knee designs at 2-year follow-up: a prospective matched-pair analysis. J Arthroplast. 2017;32:431-6.

12. Carey BW, Harty J, et al. A comparison of clinical- and patient- reported outcomes of the cemented ATTUNE and PFC sigma fixed bearing cruciate sacrificing knee systems in patients who underwent total knee replacement with both prostheses in opposite knees. J Orthop Surg Res. 2018;13:54.

13. Ali A, Lindstrand A, Nilsdotter A, Sundberg M, et al. Similar patient-reported outcomes and performance after total knee arthroplasty with or without patellar resurfacing. Acta Orthop. 2016;87:274-9.

14. Kaseb MH, Tahmasebi MN, Mortazavi SJ, Sobhan MR, Nabian MH. Comparison of clinical results between patellar resurfacing and nonresurfacing in Total knee Arthroplasty: a short term evaluation. Arch Bone Jt Surg. 2018;6(2):124-9.

15. Findlay I, Wong F, Smith C, Back D, Davies A, Ajuied A. Non-resurfacing techniques in the management of the patella at total knee arthroplasty: a systematic review and meta-analysis. Knee. 2016;23(2):191-7.

16. Gao YH, Li SQ, Yang C, Liu JG, Dong N, Qi X, et al. Favorable femoral component rotation achieved in severe varus deformity by using the gapbalancing technique. Knee. 2016;23:867-70.

17. Insall JN, Dorr LD, Scott RD, Scott WN. Rationale of the knee society clinical rating system. Clin Orthop Relat Res. 1989;248:13-4.

18. Metsna V, Vorobjov S, Lepik K, Märtson A, et al. Anterior knee pain following total knee replacement correlates with the OARSI score of the cartilage of the patella. Acta Orthop. 2014;85:427-32.

19. Helmy N, Anglin C, Greidanus NV, Masri BA, et al. To resurface or not to resurface the patella in total knee arthroplasty. Clin Orthop Relat Res. 2008; 466:2775-83.

20. Weeks CA, Marsh JD, MacDonald SJ, Graves S, Vasarhelyi EM, et al. Patellar resurfacing in Total knee Arthroplasty: a cost-effectiveness analysis. J Arthroplast. 2018:33:3412-5.

21. Maradit-Kremers H, Haque OJ, Kremers WK, Berry DJ, Lewallen DG, Trousdale RT, Sierra RJ. Is selectively not resurfacing the Patella an acceptable practice in primary Total knee Arthroplasty? J Arthroplast. 2017;32:1143-7.

22. Pilling RWD, Moulder E, Allgar V, Messner J, Sun Z, Mohsen A. Patellar resurfacing in primary Total knee replacement a meta-analysis. J Bone Joint Surg Am. 2012;94A(24):2270-8.

23. Inoue A, Arai Y, Nakagawa $S$, Inoue $H$, Yoshihara Y, Yamazoe S, Kubo T, et al. Differences in patellofemoral alignment as a result of patellar shape in cruciate-retaining total knee arthroplasty without patellar resurfacing at a minimum three-year follow-up. Knee. 2017;24:1448-53.

24. Shannak O, Palan J, Esler C, et al. A regional registry study of 216 patients investigating if patient satisfaction after total knee arthroplasty changes over a time period of five to 20 years. Knee. 2017:24:824-8.

25. Dennis DA, Kim RH, Johnson DR, Springer BD, Fehring TK, Sharma A, et al. The John Insall award: control-matched evaluation of painful patellar crepitus after total knee arthroplasty. Clin Orthop Relat Res. 2011;469:10-7.

26. Petersen W, Rembitzki IV, Brüggemann GP, Ellermann A, Best R, Koppenburg $A G$, Liebau C. Anterior knee pain after total knee arthroplasty: a narrative review. Int Orthop. 2014;38:319-28.

\section{Publisher's Note}

Springer Nature remains neutral with regard to jurisdictional claims in published maps and institutional affiliations.

Ready to submit your research? Choose BMC and benefit from:

- fast, convenient online submission

- thorough peer review by experienced researchers in your field

- rapid publication on acceptance

- support for research data, including large and complex data types

- gold Open Access which fosters wider collaboration and increased citations

- maximum visibility for your research: over $100 \mathrm{M}$ website views per year

At $\mathrm{BMC}$, research is always in progress.

Learn more biomedcentral.com/submissions 